\title{
Significance of tertiary Gleason pattern 5 in patients with Gleason score 7 after radical prostatectomy: a retrospective cohort study
}

This article was published in the following Dove Press journal: OncoTargets and Therapy

\author{
Jiakun $\mathrm{Li}^{\prime}$ \\ Yaochuan Guo ${ }^{2}$ \\ Shi Qiu' \\ Mingjing $\mathrm{He}^{\prime}$ \\ Kun Jin' \\ Xiaonan Zheng' \\ Xiang Tu' \\ Xinyang Liao' \\ Lu Yang (D)' \\ Qiang Wei $\mathbb{D}^{\prime}$ \\ 'Department of Urology, Institute of \\ Urology, Center of Biomedical Bid Data \\ and National Clinical Research Center of \\ Geriatrics, West China Hospital of \\ Sichuan University, Chengdu, Sichuan, \\ People's Republic of China; ${ }^{2}$ Department \\ of Urology, Chongqing Three Gorges \\ Central Hospital, Chongqing 404000, \\ People's Republic of China
}

Purpose: To evaluate the association between tertiary Gleason pattern 5 (TGP5) and biochemical recurrence $(\mathrm{BCR})$ in patients with prostate cancer $(\mathrm{PCa})$ with a Gleason score (GS) of 7 after radical prostatectomy (RP).

Patients and methods: This retrospective study identified 350 patients who received RP and were graded as GS $7(3+4$ or $4+3)$ at the West China Hospital from January 2009 to December 2017. Initially, the patients were divided into two groups, TGP5 absence and TGP5 presence, independent of Gleason score. We further stratified the patients by adding the Gleason score into four groups: GS 3+4, GS 3+4/TGP5, GS 4+3, and GS 4+3/TGP5. Cox proportional-hazards models were used to evaluate the association between the status of TGP5 and BCR after adjusting for the confounding factors.

Results: The risk of BCR was significantly higher in patients with TGP5 when compared to patients without TGP5 $(P=0.04, \mathrm{HR}=2.1795 \%, \mathrm{Cl}$ : $1.03-4.59)$. For patients with primary Gleason pattern 4 , the risk of BCR for patients with Gleason 4+3/TGP5 was statistically significantly higher than Gleason $4+3(P=0.04, \mathrm{HR}=2.45,95 \% \mathrm{Cl}$ : $1.04-5.76)$.

Conclusion: The TGP5 in patients with GS 7 had strong association with the risk of BCR and was an independent predictor for BCR. Further research on larger data sets is needed to confirm these findings.

Keywords: prostate cancer, biochemical recurrence, Gleason score, tertiary Gleason pattern, radical prostatectomy

\section{Introduction}

Prostate cancer $(\mathrm{PCa})$ is one of the most common malignancies among males in developed countries and is the third leading cause of cancer-related death. In the United States, PCa has the highest incidence, with an estimated 164,690 new cases in 2018. This pattern has been consistent from 1975 to 2014 . $^{1}$ In China, the percent change in the incidence of $\mathrm{PCa}$ is the highest amongst cancers in males. ${ }^{2} \mathrm{PCa}$ is a heterogeneous and multifactorial disease complicating accurate diagnosis, treatment, and assessment of prognosis of $\mathrm{PCa}$.

The Gleason system is based on the microscopic examination of prostate cancer tissue and has aided by guiding therapy and predicting outcomes. ${ }^{3-6}$ The tradition Gleason system is calculated using the first and second most prevalent histologic patterns. Adding tertiary patterns to the Gleason score has been debated as a way to increase the accuracy of the PCa prognosis. ${ }^{7-9}$ Some research has shown that tertiary Gleason pattern (TGP5) is related to disease-free survival of patients after
Qiang Wei

Department of Urology, Institute of Urology, West China Hospital of Sichuan University, No. 37, Guoxue Alley,

Chengdu, Sichuan 61004I, People's

Republic of China

Tel +86 I 898060 I425

Email weiqiang163163@163.com 
radical prostatectomy (RP). ${ }^{10-13}$ Among RP specimens, PCa with a Gleason Score (GS) 7 is the most common grade with prevalence from $30 \%$ to $50 \%$. $^{14,15}$

In patients with GS 7, some studies have indicated that TGP5 presence has worse outcomes, including biochemical recurrence (BCR) compared with those without TGP5. ${ }^{10-13,16,17}$ For this study, we assessed the impact of TGP5 on the BCR in GS 7 patients after RP at the West China Hospital.

\section{Materials and methods}

This retrospective study identified 604 patients who received RP and were diagnosed with GS $7(3+4$ or $4+3)$ in the West China Hospital. In total, 350 patients were included in this analysis (Figure 1). Data were excluded from patients without records of $\operatorname{BCR}(n=217)$ and on patients who received neoadjuvant hormone therapy (NHT) (n=37). Exclusions were not affected by the pathological diagnosis such as GS and T/N stage. A flowchart of patients who met the study's inclusion/exclusion criteria is provided as Figure S1. We collected clinicopathological data of included patients who visited the hospital between January 1, 2009 and December 31, 2017. The follow-up time was measured from the date of RP surgery to the date of emigration, date of death, or December 31, 2017, whichever was earliest. To determine the influence of TGP5 on
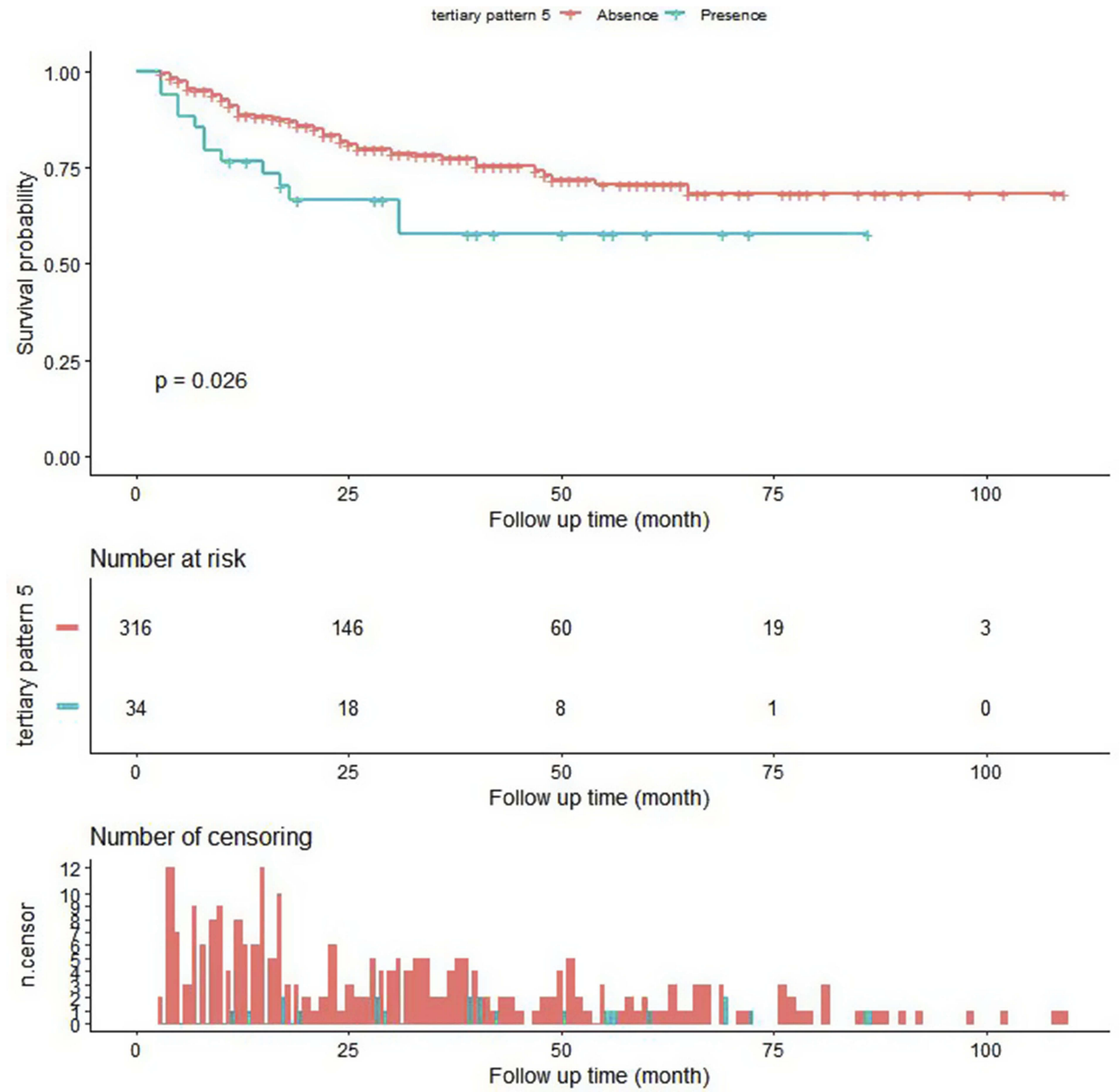

Figure I Kaplan-Meier analysis of TGP5 absence and presence regardlessly the primary Gleason pattern. The survival probability of patients with TGP5 absence and presence. 
prognosis, patients were evaluated for the presence or absence of TBP5 that was independent of the primary Gleason pattern. We identified 316 patients with absence of TGP5 and 34 patients with the presence of TGP5. We stratified our sample by the primary Gleason pattern to address heterogeneity. Our sample was stratified into 4 groups: 165 patients with primary Gleason pattern 3 with TGP5 absence (GS 3+4), 7 patients with primary Gleason pattern 3 with TGP5 presence (GS 3+4/TGP 5), 151 patients with primary Gleason pattern 4 with TGP5 absence (Gleason 4+3), and 27 patients with primary Gleason pattern 4 with TGP5 presence (Gleason 4+3/TGP 5).

The clinical and pathological characteristics included the age at surgery, surgical approach, body mass index (BMI), tumor node metastasis (TNM) stage, and serum prostate-specific antigen (PSA) level. Grading and description of surgical samples were determined by 1 or 2 experienced pathologists without knowledge of grouping. TGP5 was defined as the third component of a Gleason pattern which is higher than the primary and secondary grades with the tertiary pattern. And TGP5 visually is deemed to be less than $5 \%$ of the whole tumor. As the primary endpoint, BCR of PCa was defined by two consecutive PSA increases of $\geq 0.2 \mathrm{ng} / \mathrm{mL}$ after RP. ${ }^{18}$ The patient was seen quarterly to semiannually where follow-up PSA was performed and other clinicopathological information was collected. After 2 years, the patients were seen annually. No informed consent was needed by the Institutional Review Board West China Hospital as we analyzed de-identified patient data.

We summarized baseline characteristics with and without stratification of TGP5 status. Continuous variables using Kruskal-Wallis test were reported as means and standard deviations, while categorical variables using chisquare or Fisher's exact test were reported as counts and proportions.

We used univariate Cox regression model to evaluate the association between the status of TGP5 and BCR. Cox proportional-hazards models were used to assess the association between TGP5 and BCR controlling for other factors.

Model 1 included surgical approach, age at surgery, BMI, preoperative PSA, and biopsy GS as covariates. Model 2 included the surgical approach, age at surgery, BMI, preoperative PSA, biopsy GS, positive surgical margin (PSM), and TNM stage as covariates. We adjusted the covariates in the model using a change in the hazard ratio of $10 \%$ as a guideline. ${ }^{19}$
All analyses were performed using the statistical programs R (http://www.R-project.org, The R Foundation) and EmpowerStats (http://www.empowerstats.com, X\&Y Solutions, Inc, Boston, MA). We used stratified linear regression models for the subgroup analyses. The likelihood ratio test was used to compare the stratified samples as well as test interactions. All the statistical tests were two-sided and $P$-values of less than 0.05 were considered statistically significant.

The Institutional Review Board of West China Hospital has approved this study.

\section{Results}

A total of 350 patients with GS 7 treated with RP were analyzed in this study. The range of age was 48 to 84 years (Mean 67.92 \pm 6.75 ) and the follow-up time was from 3 to 109 months (Mean 29.05 \pm 22.98 ). The clinicopathological information is listed in Table 1. For pathological characteristics at baseline, pathological GS, biopsy GS, pathological ISUP, and BCR have differences $(P<0.05)$. There is no statistically significant difference in other clinical and pathological characteristics at baseline when stratified by GS 7 and TGP5 as well as stratified by TGP5 alone. We applied univariate Cox regression model to evaluate the unadjusted association between TBP5 and BCR. The results of the univariate Cox regression model are presented in Table S1.

In the Cox proportional-hazards models, we controlled clinical and pathological characteristics, including surgical approach, age at surgery, BMI, preoperative PSA, NHT, PSM, and TNM stage. The risk of BCR for patients with TGP5 presence was significantly higher than the absence of TGP5 ( $P=0.04, \mathrm{HR}=2.17$ 95\%, $\mathrm{Cl}: 1.03-4.59)$ independent of primary Gleason score (Table S2). BCR survival was significantly different between patients with and without TGP5 as assessed by Kaplan-Meier curves and logrank tests $(P=0.026$, Figure 1$)$.

In patients with primary Gleason pattern 4 , the risk of BCR for patients with Gleason 4+3/TGP5 was significantly higher than Gleason $4+3$ alone $(P=0.04, \mathrm{HR}=2.45$, 95\% Cl: 1.04-5.76) (Table S3). BCR survival within these strata was significant (log-rank $P=0.073$, Figure 2).

There was no significant difference in risk of BCR or BCR survival between patients with Gleason 3+4/TGP 5 compared to patients with Gleason 3+4 (Table S3 and Figure 3).

Hierarchical regression analysis for subgroups showed a stronger association between TGP5 and BCR among subgroups of patients with PSA $\geq 20 \mathrm{ng} / \mathrm{mL} \quad(p=0.02)$, pathological T stage $=2 \mathrm{c}(P=0.04)$, pathological $\mathrm{N}$ stage $=0$ 
Table I Baseline characteristics of participants

\begin{tabular}{|c|c|c|c|c|c|}
\hline Tertiary pattern 5 & Absence & Presence & $P$-value & Mean+SD & $\mathbf{N}(\%)$ \\
\hline $\mathrm{N}$ & 316 & 34 & & & \\
\hline Age at surgery (year, Mean+SD) & $67.78 \pm 6.76$ & $69.26 \pm 6.64$ & 0.202 & $67.92 \pm 6.75$ & \\
\hline Postoperative hospital stay (day, Mean+SD) & $7.36 \pm 3.67$ & $8.15 \pm 4.67$ & 0.069 & $7.44 \pm 3.78$ & \\
\hline BMI (Mean+SD) & $23.79 \pm 2.79$ & $23.53 \pm 2.14$ & 0.787 & $23.76 \pm 2.74$ & \\
\hline Intraoperative bleeding (mL, Mean+SD) & $305.63 \pm 258.64$ & $230.75 \pm 136.46$ & 0.183 & $298.36 \pm 250.27$ & \\
\hline Preoperative PSA (ng/mL, Mean+SD) & $17.95 \pm|6.3|$ & $21.40 \pm 20.11$ & 0.415 & $18.28 \pm 16.72$ & \\
\hline Follow-up time (month, Mean+SD) & $28.88 \pm 23.02$ & $30.59 \pm 22.90$ & 0.607 & $29.05 \pm 22.98$ & \\
\hline Surgical approach (N, \%) & & & 0.154 & & \\
\hline Open RRP & $70(22.15 \%)$ & $8(23.53 \%)$ & & & 78 (22.29\%) \\
\hline LRP & II 2 (35.44\%) & $17(50.00 \%)$ & & & $129(36.86 \%)$ \\
\hline RARP & 134 (42.4I\%) & $9(26.47 \%)$ & & & $143(40.86 \%)$ \\
\hline Pathological GS (N, \%) & & & $<0.001$ & & \\
\hline $3+4$ & 165 (52.22\%) & 7 (20.59\%) & & & $172(49.14 \%)$ \\
\hline $4+3$ & $15 \mathrm{I}(47.78 \%)$ & 27 (79.4I\%) & & & 178 (50.86\%) \\
\hline Biopsy GS (N, \%) & & & $<0.001$ & & \\
\hline $3+3$ & 48 (15.19\%) & 4 (II.76\%) & & & $52(14.86 \%)$ \\
\hline $3+4$ & $156(49.37 \%)$ & $9(26.47 \%)$ & & & $165(47.14 \%)$ \\
\hline $3+5$ & $0(0.00 \%)$ & $2(5.88 \%)$ & & & $2(0.57 \%)$ \\
\hline $4+3$ & $88(27.85 \%)$ & $13(38.24 \%)$ & & & I0I (28.86\%) \\
\hline $4+4$ & 18 (5.70\%) & $3(8.82 \%)$ & & & $21(6.00 \%)$ \\
\hline $4+5$ & $2(0.63 \%)$ & $2(5.88 \%)$ & & & $4(1.14 \%)$ \\
\hline $5+3$ & $0(0.00 \%)$ & I (2.94\%) & & & I (0.29\%) \\
\hline $5+4$ & I (0.32\%) & $0(0.00 \%)$ & & & I (0.29\%) \\
\hline NA & $3(0.95 \%)$ & $0(0.00 \%)$ & & & $3(0.86 \%)$ \\
\hline Pathological ISUP (N, \%) & & & $<0.001$ & & \\
\hline 2 & 165 (52.22\%) & 7 (20.59\%) & & & $172(49.14 \%)$ \\
\hline 3 & I5I (47.78\%) & 27 (79.4I\%) & & & $178(50.86 \%)$ \\
\hline Intraoperative blood transfusion ( $\mathrm{N}, \%)$ & & & 0.239 & & \\
\hline No & $296(93.67 \%)$ & $34(100.00 \%)$ & & & $330(94.29 \%)$ \\
\hline Yes & $20(6.33 \%)$ & $0(0.00 \%)$ & & & $20(5.71 \%)$ \\
\hline Damico grade $(\mathrm{N}, \%)$ & & & 0.505 & & \\
\hline Low risk & 7 (2.22\%) & I (2.94\%) & & & 8 (2.29\%) \\
\hline Medium risk & 119 (37.66\%) & $10(29.41 \%)$ & & & $129(36.86 \%)$ \\
\hline High risk & $190(60.13 \%)$ & $23(67.65 \%)$ & & & $213(60.86 \%)$ \\
\hline $\mathrm{T}$ stage $(\mathrm{N}, \%)$ & & & 0.121 & - & \\
\hline $\mathrm{T} 2 \mathrm{a}$ & $48(16.44 \%)$ & $3(10.00 \%)$ & & & $5 \mathrm{I}(15.84 \%)$ \\
\hline $\mathrm{T} 2 \mathrm{~b}$ & $90(30.82 \%)$ & $9(30.00 \%)$ & & & 99 (30.75\%) \\
\hline $\mathrm{T} 2 \mathrm{c}$ & $|3|(44.86 \%)$ & $12(40.00 \%)$ & & & | 43 (44.4I\%) \\
\hline T3a & $13(4.45 \%)$ & $2(6.67 \%)$ & & & $15(4.66 \%)$ \\
\hline T3b & $10(3.42 \%)$ & 4 (13.33\%) & & & 14 (4.35\%) \\
\hline $\mathrm{N}$ stage $(\mathrm{N}, \%)$ & & & 0.195 & & \\
\hline No & $303(95.89 \%)$ & $31(91.18 \%)$ & & & $334(95.43 \%)$ \\
\hline $\mathrm{NI}$ & $13(4.11 \%)$ & 3 (8.82\%) & & & 16 (4.57\%) \\
\hline$M$ stage $(\mathrm{N}, \%)$ & & & I & & \\
\hline Mo & $306(96.84 \%)$ & $33(97.06 \%)$ & & & $339(96.86 \%)$ \\
\hline MIb & $10(3.16 \%)$ & I (2.94\%) & & & II (3.14\%) \\
\hline
\end{tabular}

(Continued) 
Table I (Continued).

\begin{tabular}{|l|l|l|l|l|l|}
\hline Tertiary pattern 5 & Absence & Presence & P-value & Mean+SD & N (\%) \\
\hline PSM (N, \%) & & & 0.653 & \\
No & $251(80.19 \%)$ & $26(76.47 \%)$ & & \\
Yes & $62(19.81 \%)$ & $8(23.53 \%)$ & & $70(79.83 \%)$ \\
\hline BCR (N, \%) & & & 0.013 & & \\
No & $257(81.33 \%)$ & $21(61.76 \%)$ & & $278(79.43 \%)$ \\
Yes & $59(18.67 \%)$ & $13(38.24 \%)$ & & $72(20.57 \%)$ \\
\hline
\end{tabular}

Abbreviations: RRP, retropubic radical prostatectomy; LRP, laparoscopic radical prostatectomy; RARP, robotic-assisted laparoscopic radical prostatectomy; ISUP, International Society of Urologic Pathology grade; PSM, positive surgical margin.

$(P=0.03)$, or pathological M sge $=0$ ta $(P=0.03)$ (Table S4). characteristics was not statistically significant. The test Stratified analysis in other clinical and pathological for interactions was showed in Table S5.

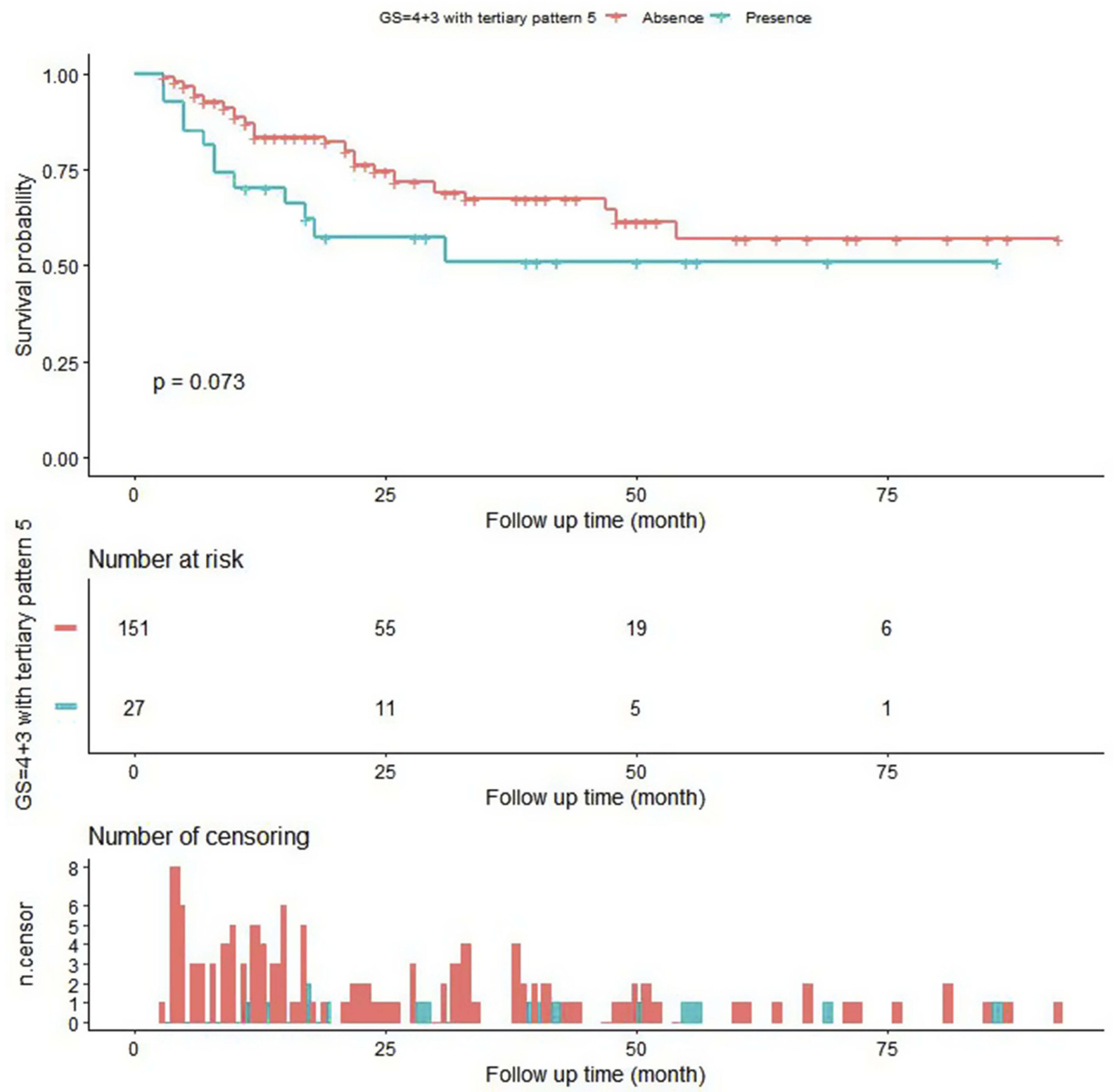

Figure 2 Kaplan-Meier analysis of GS $4+3$ and GS 4+3/TGP5. The survival probability of patients with GS 4+3 and GS 4+3/TGP5. The log-rank $p=0.073$. 


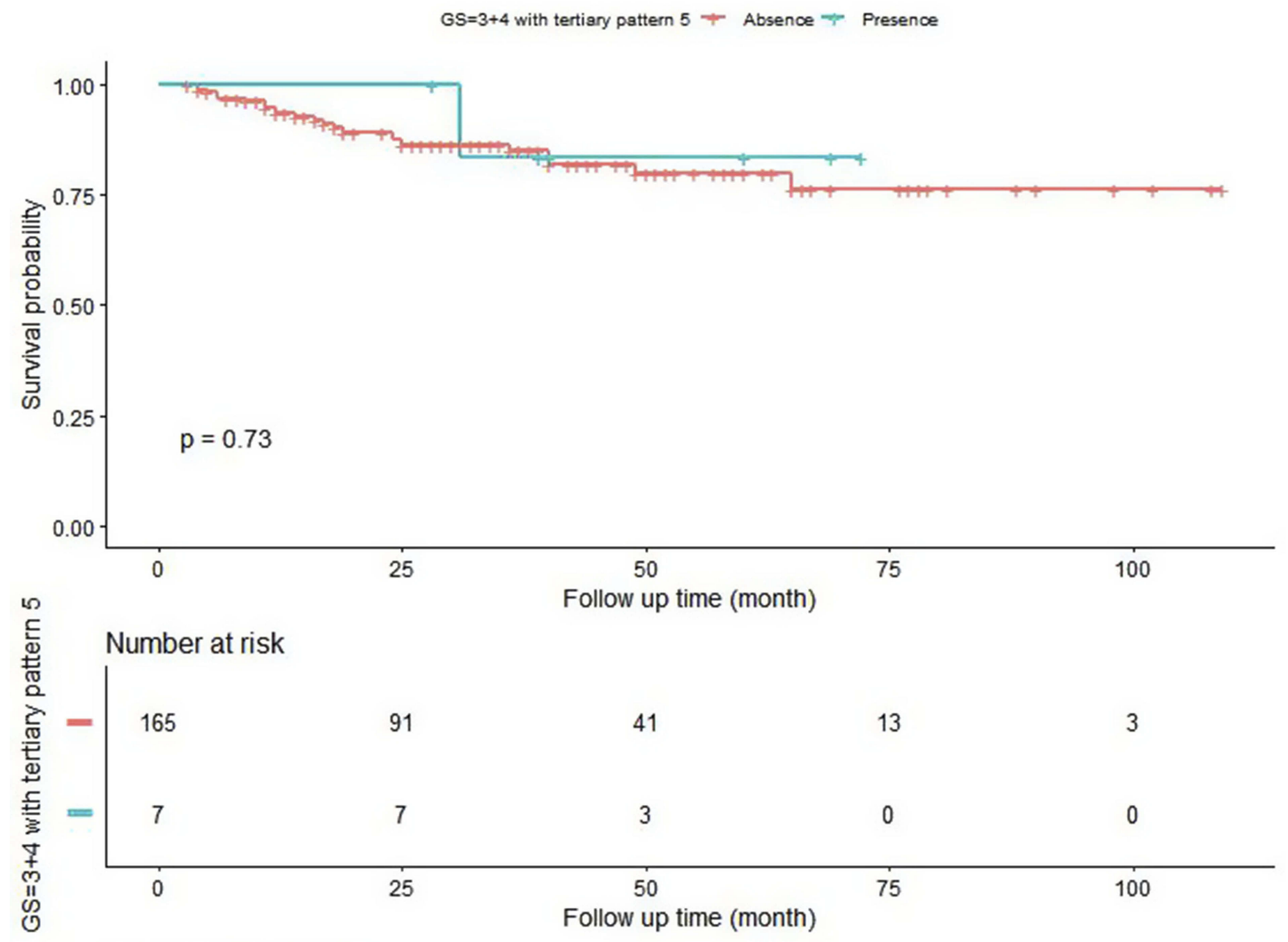

Number of censoring

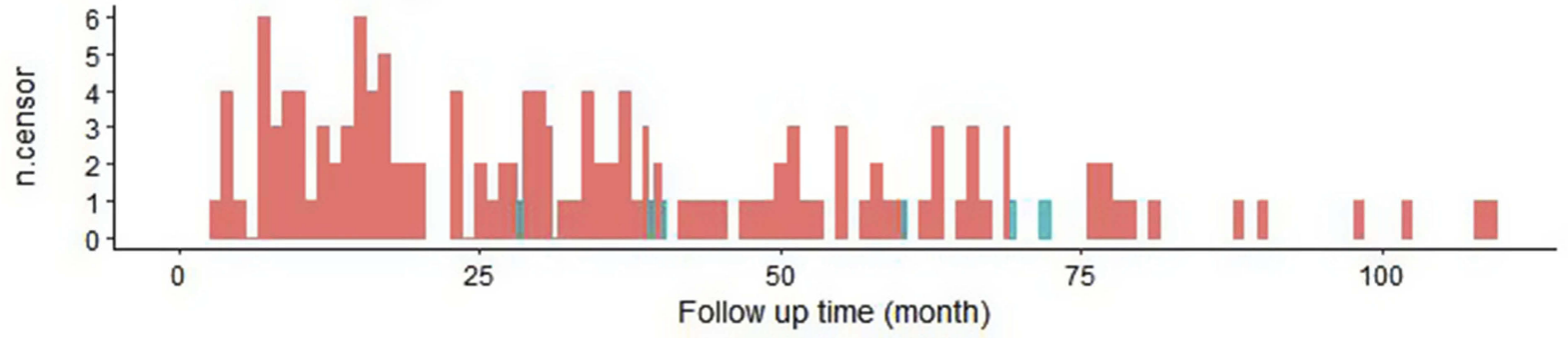

Figure 3 Kaplan-Meier analysis of GS3+4 and GS3+4/TGP5. The survival probability of patients with GS3+4 and GS3+4/TGP5. There was no significant difference in BCR survival between patients with Gleason 3+4/TGP 5 compared to patients with Gleason 3+4 (log-rank $P=0.73$ ).

\section{Discussion}

Although the relevance of TGP5 to more advanced PCa has reached consensus, the effects of TCG on BCR are still controversial. Some studies ${ }^{20-24}$ reported TGP is an independent predictor of BCR, while other studies ${ }^{25,26}$ did not find a significant association.

In this study, we identified patients with GS 7 and found that TGP5 was associated with BCR in patients after RP, confirming the results of previous studies. Overall, we showed a worse prognosis with individuals with TGP5, and this relationship persisted regardless of the primary Gleason pattern $(P<0.05)$. When stratifying by primary Gleason pattern, there was a significant difference showing Gleason $4+3 /$ TGP5 had a worse prognosis than Gleason $4+3(P<0.05)$.

Even a small amount of TGP 5 leads to serious deterioration of the prognosis and TGP 5 is relative with the further clinical expansion negatively impacts prognosis. ${ }^{16}$ Özsoy M et al reported that TGP5 increased the risk of BCR within the same GS group, which including patients with GS $7(4+3)$ and $(3+4) .{ }^{27}$ Sim et al also reported that TGP was significantly associated with increased risk of BCR in GS 7 groups in 1100 cases. $^{21}$ Baras et al, stated that, in patients with GS 7, the effect of TGP5 in pattern $4+3$ was greater than pattern 
$3+4$ with respect to $\mathrm{BCR},{ }^{28}$ which is consistent with our findings. Jang et al reported the presence of TGP5 is an independent predictor of BCR in patients with GS $7^{29}$ presence of TGP5 in patients with GS 7 increased the risk of BCR to mimic the rate of GS 8. Trock et al found that patients with GS $7(4+3)$ and TGP5 had the same risk of BCR in patients with GS $8(4+4) .{ }^{20}$ Moul et $\mathrm{al}^{6}$ found patients with GS 7 and higher-grade tertiary patterns had the same risk of BCR with patients with GS8. Sauter et al even defined an integrated quantitative GS by considering TGP5 to reflect their finding that TGP5 had powerful prognostic impact in patients with GS $7 .^{23}$

Our study found a significant association of the presence of TGP5 and the risk of BCR in patients with GS 7 had a significant association with the risk of BCR, supporting inclusion of TGP5 in the evaluation of patients with PCa. They may consider TGP5 when calculating the risk of BCR for patients with GS 7 after PR. The evidence, including this study, suggests that the current Gleason grading system should be updated to include TGP5.

Our study is the first research of this topic to analyze the patients in this region of China, where there is an estimated incidence $(60.3 \%)$ of $\mathrm{PCa}$ and associated mortality $(26.6 \%)$ within the 4,292,000 invasive cancer cases in $2015 .^{2}$

There were several limitations of our study including relatively small sample size. Our data were collected from clinical records at the West China Hospital. Although it was the top medical institution for the western part of China, it is only a single institution, and our results may not be generalizable. We found interesting non-significant differences in the stratified analysis result, but could not make conclusions because of our sample size. Further studies with larger sample sizes can more fully test these associations.

We are constructing a database which includes additional data and more extended follow-up information, providing a valuable resource to further study the association between TGP5 in patients with GS 7 and the risk of BCR. Finally, the Gleason grading system should consider including TGP5 information to improve the assessment of prognosis in patients with $\mathrm{PCa}$.

\section{Conclusion}

We found a significant association between TGP5 and BCR in patients with GS 7 after RP. The TGP5 in patients with GS 7 had strong association with the risk of BCR and it was an independent predictor for BCR. This result was stronger in patients with GS $7(4+3)$. Further research with larger dataset is needed to confirm these findings.

\section{Acknowledgments}

The poster's abstract was published in "Paper Abstracts" in the Journal of clinical oncology, hyperlink with DOI: https://ascopubs.org/doi/abs/10.1200/JCO.2019.37.7_suppl. 117. The authors are thankful for the patients and the colleagues at the Department of Urology, Institute of Urology, West China Hospital who participated in this study.

The authors gratefully thank Dr. Changzhong Chen, Chi Chen, and Xin-Lin Chen (EmpowerStats X\&Y Solutions, Inc., Boston, MA) for providing statistical methodology consultation. This work was supported by National Key Research and Development Program of China (SQ2017YFSF090096), National Natural Science Foundation of China (81770756) and Sichuan Science and Technology Program (2017HH0063).

\section{Disclosure}

The authors report no conflicts of interest in this work.

\section{References}

1. Chen W, Zheng R, Baade PD, et al. Cancer statistics in China, 2015. CA Cancer J Clin. 2016;66(2):115-132. doi:10.3322/caac.21338

2. Mottet N, Bellmunt J, Briers E, et al. EAU-ESTRO-SIOG guidelines on prostate cancer. Follow-up: after local treatment. Eur Urol. 2017;7 (2):3.

3. Epstein JI, Partin AW, Sauvageot J, et al. Prediction of progression following radical prostatectomy. A multivariate analysis of 721 men with long-term follow-up. Am J Surg Pathol. 1996;20:286-292. doi:10.1097/00000478-199603000-00004

4. Partin AW, Kattan MW, Subong EN, et al. Combination of prostatespecific antigen, clinical stage, and Gleason score to predict pathological stage of localized prostate cancer. A multi-institutional update. JAMA. 1997;277:1445-1451.

5. Catalona WJ, Smith DS. Cancer recurrence and survival rates after anatomic radical retropubic prostatectomy for prostate cancer: intermediate-term results. J Urol. 1998;160:2428. doi:10.1097/00005392199809020-00019

6. Moul JW, Connelly RR, Lubeck DP, et al. Predicting risk of prostate specific antigen recurrence after radical prostatectomy with the Center for Prostate Disease Research and Cancer of the Prostate Strategic Urologic Research Endeavor databases. $J$ Urol. 2001;166:1322. doi:10.1016/S0022-5347(05)65761-8

7. Gleason DF, Mellinger GT. Prediction of prognosis for prostatic adenocarcinoma by combined histological grading and clinical staging. J Urol. 1974;111:58-64. doi:10.1016/S0022-5347(17)59889-4

8. Gleason DF. Classification of prostatic carcinomas. Cancer Chemother Rep. 1966;50:125-128.

9. Polat T, Bas E, Bozkurt S, et al. Presence of high grade tertiary Gleason pattern upgrades the Gleason sum score and is inversely associated with biochemical recurrence-free survival. The Journal of Urology. 185(4):e80-e81. doi:10.1016/j.juro.2011. 02.264

10. Rasiah KK, Stricker PD, Haynes AM, et al. Prognostic significance of Gleason pattern in patients with Gleason score 7 prostate carcinoma. Cancer. 2003;98:2560. doi:10.1002/cncr.11850 
11. Hattab EM, Koch MO, Eble JN, Lin H, Cheng L. Tertiary Gleason pattern 5 is a powerful predictor of biochemical relapse in patients with Gleason score 7 prostatic adenocarcinoma [discussion 1699]. $J$ Urol. 2006;175:1695-1699. doi:10.1016/S0022-5347(05)00998-5

12. Mosse CA, Magi-Galluzi C, Tsuzuki T, Epstein JI The prognostic significance of tertiary Gleason pattern 5 in radical prostatectomy specimens. Am J Surg Pathol. 2004;28:394.

13. Pan CC, Potter SR, Partin AW, Epstein JI. The prognostic significance of tertiary Gleason patterns of higher grade in radical prostatectomy specimens: a proposal to modify the Gleason grading system. Am J Surg Pathol. 2000;24:563. doi:10.1097/00000478200004000-00011

14. Rasiah KK, Stricker PD, Haynes AM, et al. Prognostic significance of Gleason pattern in patients with Gleason score 7 prostate carcinoma. Cancer. 2003;98:2560. doi:10.1002/cncr.11850

15. Tefilli MV, Gheiler EL, Tiguert R, et al. Should Gleason score 7 prostate cancer be considered a unique category? Urology. 1999;53:372. doi:10.1016/S0090-4295(98)00479-8

16. Krech T, Lutz F, Lennartz M, et al. Integrating tertiary Gleason 5 patterns into quantitative gleason grading in prostate biopsies and prostatectomy specimens. Eur Urol. 2018;73:674-683. doi:10.1016/j. eururo.2017.01.015

17. Whittemore DE, Hick EJ, Carter MR, et al. Significance of tertiary Gleason pattern 5 in Gleason score 7 radical prostatectomy specimens. J Urol. 2008;179(February):516-522. doi:10.1016/j.juro.2007.09.085

18. Boccon-Gibod L, Djavan B, Hammerer P, et al. Management of prostate-specific antigen relapse in prostate cancer: a European Consensus. Int J Clin Pract. 2004;58:382. doi:10.1111/j.13685031.2004.00184.x

19. Kernan WN, Viscoli CM, Brass LM, et al. Phenylpropanolamine and the risk of hemorrhagic stroke. N Engl J Med. 2000;343:1826-1832. doi:10.1056/NEJM200012213432501

20. Trock BJ, Guo CC, Gonzalgo ML, Magheli A, Loeb S, Epstein JI. Tertiary Gleason patterns and biochemical recurrence after prostatec tomy: proposal for a modified gleason scoring system. J Urol. 2009;182:1364-1370. doi:10.1016/j.juro.2009.06.048
21. Sim HG, Telesca D, Culp SH, et al. Tertiary Gleason pattern 5 in Gleason 7 prostate cancer predicts pathological stage and biochemical recurrence. $J$ Urol. 2008;179:1775-1779. doi:10.1016/j. juro.2008.01.016

22. Turker P, Bas E, Bozkurt S, et al. Presence of high grade tertiary Gleason pattern upgrades the Gleason sum score and is inversely associated with biochemical recurrence-free survival. Urol Oncol. 2013;31:93-98. doi:10.1016/j.urolonc.2010.10.009

23. Sauter G, Clauditz T, Steurer $\mathrm{S}$, et al. Integrating tertiary Gleason 5 patterns into quantitative Gleason grading in prostate biopsies and prostatectomy specimens. Eur Urol. 2018;73:674-683. doi:10.1016/j. eururo.2017.01.015

24. Borhan W, Epstein JI. Significance of Gleason score 7 with tertiary pattern 5 at radical prostatectomy. Urology. 2016;100:175-179. doi:10.1016/j.urology.2016.10.035

25. van Oort IM, Schout BM, Kiemeney LALM, Hulsbergen CA, Witjes JA. Does the tertiary Gleason pattern influence the PSA progressionfree interval after retropubic radical prostatectomy for organ-confined prostate cancer? Eur Urol. 2005;48:572-576. doi:10.1016/j. eururo.2005.06.003

26. Hashine K, Yuasa A, Shinomori K, Shirato A, Ninomiya I, Teramoto N. Tertiary Gleason pattern 5 and oncological outcomes after radical prostatectomy. Jpn J Clin Oncol. 2011;41:571-576. doi:10.1093/jjco/ hyq 250

27. Özsoy M, Andrea DD, Moschini M, et al. Tertiary Gleason pattern in radical prostatectomy specimens is associated with worse outcomes than the next higher Gleason score group in localized prostate cancer. Urol Oncol Semin Orig Investig. 2018;36(4):158.e1-158.e6. doi:10.1016/j.urolonc.2017.12.003

28. Baras AS, Nelson JB, Han M, Parwani AV, Epstein JI. The effect of limited (tertiary) Gleason pattern 5 on the new prostate cancer grade groups. Hum Pathol. 2017;63:27-32. doi:10.1016/j.humpath.2016.12.008

29. Jang WS, Yoon CY, Kim MS, et al. The prognostic role of tertiary Gleason pattern 5 in a contemporary grading system for prostate cancer. Prostate Cancer Prostatic Dis. 2017;2016:93-98. doi:10.1038/pcan.2016.55

\section{Publish your work in this journal}

OncoTargets and Therapy is an international, peer-reviewed, open access journal focusing on the pathological basis of all cancers, potential targets for therapy and treatment protocols employed to improve the management of cancer patients. The journal also focuses on the impact of management programs and new therapeutic agents and protocols on patient perspectives such as quality of life, adherence and satisfaction. The manuscript management system is completely online and includes a very quick and fair peer-review system, which is all easy to use. Visit http://www.dovepress.com/ testimonials.php to read real quotes from published authors. 